\title{
Involvement of MrgprC in Electroacupuncture Analgesia for Attenuating CFA-Induced Thermal Hyperalgesia by Suppressing the TRPV1 Pathway
}

\author{
Ying-jun Liu $\mathbb{D}^{\mathbb{D}}$, Xiao-xi Lin $\left(\mathbb{D}\right.$, Jian-qiao Fang $\mathbb{D}^{\mathbb{D}}$, and Fang Fang $\mathbb{D}$ \\ Department of Neurobiology and Acupuncture Research, The Third Clinical Medical College, \\ Zhejiang Chinese Medical University, Hangzhou 310053, China \\ Correspondence should be addressed to Jian-qiao Fang; fangjianqiao7532@163.com and Fang Fang; 13185096105@163.com
}

Received 14 October 2017; Revised 22 December 2017; Accepted 4 January 2018; Published 12 February 2018

Academic Editor: Shu-Ming Wang

Copyright (C) 2018 Ying-jun Liu et al. This is an open access article distributed under the Creative Commons Attribution License, which permits unrestricted use, distribution, and reproduction in any medium, provided the original work is properly cited.

\begin{abstract}
Mas-related G-protein-coupled receptor C (MrgprC) plays an important role in modulating chronic inflammatory pain. Electroacupuncture (EA) has a satisfactory analgesic effect on chronic pain. This study aimed to investigate the involvement of MrgprC and its transient receptor potential vanilloid 1 (TRPV1) pathway in EA analgesia in chronic inflammatory pain. Chronic inflammatory pain was induced by subcutaneously injecting complete Freund's adjuvant (CFA) into the left hind paw. EA $(2 / 100 \mathrm{~Hz})$ stimulation was administered. MrgprC siRNAs were intrathecally administered to inhibit MrgprC expression, and bovine adrenal medulla 8-22 (BAM8-22) was used to activate MrgprC. The mechanical allodynia was decreased by EA significantly since day 3. The piled analgesic effect of EA was partially blocked by 6 intrathecal administrations of MrgprC siRNA. Both EA and BAM8-22 could downregulate the expression of TRPV1 and PKC in both the DRG and the SCDH. Both EA and BAM8-22 could also decrease the TRPV1 translocation and p-TRPV1 level in both the DRG and the SCDH. The effects of EA on PKCE, TRPV1 translocation, and p-TRPV1 in both the DRG and the SCDH were reversed by MrgprC siRNA. The results indicated that MrgprC played crucial roles in chronic pain modulation and was involved in EA analgesia partially through the regulation of TRPV1 function at the DRG and SCDH levels.
\end{abstract}

\section{Introduction}

Chronic inflammatory pain is a kind of refractory disease, and the prevalence rate in developing countries is higher than developed countries, with approximately $33 \%$ in the general adult population and $56 \%$ in the elderly population [1]. Cyclooxygenase-2 (COX-2), tumor necrosis factor- $\alpha$ (TNF$\alpha$ ), substance $\mathrm{P}(\mathrm{SP})$, and other neurotransmitters that are stimulated by sustained inflammation activate ion channels such as transient receptor potential vanilloid 1 (TRPV1) and acid-sensing ion channels (ASICs) and then induce peripheral pain sensitization [2]. The ongoing input conveyed from peripheral neurons overexcites spinal nociceptive neurons, and finally central sensitization is induced. On the other hand, nociceptive information transferred from the periphery to the spinal cord can also be modulated in peripheral sensory neurons, especially in the dorsal root ganglia (DRG), through the activation of the opioid system $[3,4]$.
Electroacupuncture (EA), a commonly used acupuncture method, has been widely promoted for alleviating chronic inflammatory pain [5]. Previous studies of EA analgesia mainly focused on spinal and supraspinal mechanisms. However, we found that local acupuncture can achieve better analgesic effects than distal acupuncture for treatment of chronic pain in clinics [6]. In addition, it is difficult to explain that local injection of anesthetic procaine showed inhibition of the EA analgesic effect [7]. All findings above remain to be explained. A previous study showed that nociceptive response stimulated by complete Freund's adjuvant (CFA) can be reduced by treatment with EA with dilatational $100 \mathrm{~Hz}$ and $2 \mathrm{~Hz}$ alternating frequencies $(2 / 100 \mathrm{~Hz})$ on ST36 [8], which is related to the peripheral endogenous opioid system [9].

Mas-related G protein-coupled receptor C (MrgprC) is an atypical opioid receptor activated by opioids. The unique endogenous ligand of MrgprC is bovine adrenal medulla 22 peptide (BAM22), a degradation product of proenkephalin. 
BAM22 can be combined with $\mu, \delta$, and $\kappa$ opioid receptors, but its activation on MrgprC cannot be antagonized by naloxone [10]. MrgprC is dramatically expressed in medium and small neurons of the DRG and trigeminal ganglion [11, 12], and then a section of MrgprC transports to lamina II of the spinal cord dorsal horn (SCDH) [11]. The highly restricted distribution of MrgprC makes it a potential therapeutic target with fewer central side effects [13]. Recent studies have shown that MrgprC plays an important role in modulating chronic inflammatory pain $[10-12,14]$ by inhibiting the increased expression of neuronal nitric oxide synthase (nNOS), calcitonin gene related peptide (CGRP), and c-fos in the DRG or $\mathrm{SCDH}$ [15]. Our previous studies have shown that MrgprC may be involved in the analgesic effect of EA [16], but the specific mechanism is unclear.

TRPV1 is an integration factor of nociceptive signals (heat, cold, and inflammation factors) in the DRG, and it is one of the key mechanisms that induce the thermal pain sensitization [17]. Both bilateral and unilateral EA at ST36 can inhibit the expression of TRPV1 in the DRG and SCDH under conditions of chronic inflammatory pain induced by CFA, which can also trigger reduction of a series of pain-sensitive molecules such as phosphorylated extracellular regulated protein kinases (p-ERK), phosphorylated cAMP-response element binding protein ( $\mathrm{p}$-CREB), phosphorylated P38 ( $\mathrm{p}$ p38), and others [18]. However, limited data have been obtained on regulatory effects of EA on TRPV1 in the spinal cord and how TRPV1 is regulated by EA in chronic inflammatory pain. TRPV1 has been demonstrated to be a major downstream target in MrgprC-activating signaling pathways in acute inflammatory pain $[14,19,20]$, and it is mediated by PKC [21]. MrgprC might be involved in the modulation of pain sensitization through the PKC/TRPV1 pathway in the DRG and SCDH.

In this study, to observe MrgprC in the EA analgesic effect, we established chronic inflammatory pain by subcutaneously injecting CFA and blocking MrgprC expression by intrathecally administrating MrgprC small interfering RNA (siRNA). By applying intrathecal administration of bovine adrenal medulla 8-22 (BAM8-22), a C-terminal artificial hydrolytic fragment of BAM22, the MrgprC-specific agonist, as a control, we observed TRPV1 expression, TRPV1 translocation from the cytoplasm to the PM, and the level of p-TRPV1 mediated by PKC in the DRG and SCDH to investigate the possible mechanisms of MrgprC modulation of peripheral pain sensitization by EA.

\section{Materials and Methods}

2.1. Animals. Adult male Sprague-Dawley rats (280-300 g) were purchased from the BK (Shanghai Sippr-BK Laboratory Animal Co. Ltd., China) and housed individually at $25 \pm 2{ }^{\circ} \mathrm{C}$ under a $12 \mathrm{~h}$ alternating light-dark cycle with food and water ad libitum. The treatment of animals was strictly performed in accordance with the guidelines of the regulations of the State Science and Technology Commission for the Care and Use of Laboratory Animals (State Science and Technology Commission Number 2, 1988). All efforts were made to minimize animal suffering and to reduce the number of animals used in this study.
2.2. Intrathecal Catheter Implantation. As Yaksh and Rudy previously reported [22], rats were implanted with indwelling catheters into the lumbar vertebra subarachnoid. Briefly, a rat was anesthetized with $7.5 \%$ sodium chloral hydrate. A polyethylene catheter (PE, OD $0.50 \mathrm{~mm} \times$ I.D $0.25 \mathrm{~mm}$, RWD Life Science Co., Ltd., China) was inserted into the subarachnoid space from the back of L5-L6 lumbar intervertebral till its tip was positioned at the L4-L5 spinal cord. The other end of the catheter was anchored in the back of the lumbar. A successful indication was a temporary motor paralysis of the bilateral lower limbs induced by intrathecal lidocaine (0.1 g/5 mL; Otsuka Pharmaceutical Co., Ltd., China) $24 \mathrm{~h}$ after catheter implantation. The rats were allowed to recover for 5-6 days. Only rats without evidence of neurologic deficits were chosen for the experiment.

2.3. Establishment of Model and Experimental Groups. Inflammatory pain was induced by subcutaneously injecting (sc) $0.1 \mathrm{~mL}$ CFA (Sigma, St. Louis, USA) into the left hinder paw. The rats were divided randomly into 5 groups: normal group, model group, EA + control siRNA group, EA + MrgprC siRNA group, and BAM8-22 group ( $n=8$ per group). The normal group was injected with $0.1 \mathrm{~mL}$ saline, while the other 4 groups received CFA injection.

2.4. Treatments. As Ndong and his colleagues reported [14], MrgprC siRNA sequences 5'-CAUGUCAGCUAUUAUAUGUt-ps-t- $3^{\prime}$ and $3^{\prime}$-t-ps-tGUACAGUCGAUAAUAUACA- $5^{\prime}$ (GenePharma Co., Ltd., China) and mismatch control siRNA sequences $5{ }^{\prime}$-CAAGUUAUCUAGUAAUUAUa-ps-t- $3^{\prime}$ and $3^{\prime}$-t-ps-aGUUCAAUAGAUCAUUAAUA$5^{\prime}$ (GenePharma Co., Ltd., China) were used. In these sequences, " $\mathrm{t}$ " and " $\mathrm{a}$ " represented $2^{\prime}$-O-methyluridine and $2^{\prime}$-O-methyladenine, respectively, and "ps" was a phosphorothioate linkage. SiRNAs were dissolved in transfection regent i-Fect ${ }^{\mathrm{TM}}$ (Neuromics, USA) at a concentration of $5 \mu \mathrm{g} / \mu \mathrm{L}$ and were applied $1 \mathrm{~d}, 2 \mathrm{~d}, 3 \mathrm{~d}, 4 \mathrm{~d}, 5 \mathrm{~d}$, and $6 \mathrm{~d}$ after CFA injection. BAM8-22 (10 nmol, $10 \mu \mathrm{L}$, Abcam Co., Ltd., USA), a selective agonist of MrgprC, was administered intrathecally $2 \mathrm{~d}$ and $5 \mathrm{~d}$ after CFA injection.

As described previously [23], rats were immobilized loosely by cloth cover. Aseptic acupuncture needles $(0.25 \mathrm{~mm}$ in diameter) were inserted into the bilateral Zusanli (ST36, $5 \mathrm{~mm}$ lateral to the anterior tubercule of the tibia) and Kunlun (BL60, at the ankle joint level and between the tip of the external malleolus and tendon calcaneus) since both acupoints were reported to be adept in treating inflammatory pain [24]. The terminals of unilateral couple needles in ST36 and BL60 were connected to electrodes from the HANS Acupuncture point nerve stimulator (LH-202H, Huawei Co., Ltd., China). No anesthesia was used during the EA treatment. The $2 / 100 \mathrm{~Hz}$ EA (alternation of $2 \mathrm{~Hz}$ and $100 \mathrm{~Hz}$, $0.2 \mathrm{~ms}$ pulse width) was applied at an intensity of $1 \mathrm{~mA}$ for the first $15 \mathrm{~min}$ and $2 \mathrm{~mA}$ for another $15 \mathrm{~min}$ for a total of $30 \mathrm{~min}$ once per day in the EA + control siRNA and EA + MrgprC siRNA groups.

2.5. Measurement of Thermal Hyperalgesia. All tests were performed during the light phase. To observe thermal hyperalgesia, the paw withdrawal latency $(\mathrm{PWL}, \mathrm{s})$ was measured 
with a plantar test apparatus (37370, UGO Basile, Italy). Room temperature was maintained at $25 \pm 2^{\circ} \mathrm{C}$ during the test. Rats were adapted in a plastic chamber $(69 \mathrm{~cm} \times 17 \mathrm{~cm} \times$ $14 \mathrm{~cm}$ ) on an elevated glass plate for $30 \mathrm{~min}$. The radiant heat source was positioned under the center of the hind paw, and the withdrawal latency was recorded. The intensity of the light source was adjusted to obtain the PWL of normal animals within approximately $10 \mathrm{~s}$ to $15 \mathrm{~s}$. A cut-off time of $20 \mathrm{~s}$ was set to protect the animal from tissue damage. PWLs were measured $1 \mathrm{~d}$ before CFA injection and $1 \mathrm{~d}, 2 \mathrm{~d}, 3 \mathrm{~d}, 4 \mathrm{~d}$, $5 \mathrm{~d}$, and $6 \mathrm{~d}$ after CFA injection (chronic inflammatory pain phase). At any test point, PWL was measured 3 times at 5minute intervals and averaged values were calculated.

\subsection{Total Protein and Cytoplasmic and Plasma Membrane} (PM) Proteins Extraction. Ipsilateral DRG and SCDH from rats were harvested and homogenized in RIPA lysis buffer with added $1 \mathrm{mM}$ PMSF and phosphatase inhibitor (Na3VO4 and $\mathrm{NaF}$ ). The lysate was centrifuged at $14,000 \mathrm{~g}$ for $5 \mathrm{~min}$ at $4^{\circ} \mathrm{C}$ and the supernatant was collected as total protein. Cytoplasmic and PM proteins were fractionated following the manufacturer's protocol of the Plasma Membrane Protein Extraction Kit (BioVision Inc., USA) [25]. To extract cytoplasmic protein, ipsilateral DRG and SCDH were lysed in Homogenization Buffer on ice by inserting and rotating for approximately $70-80$ times until $70 \% \sim 80 \%$ of the neurons were lysed under light microscopy. Then, the lysate was centrifuged at $700 \times \mathrm{g}$ for $10 \mathrm{~min}$ at $4^{\circ} \mathrm{C}$ to remove nuclei and unlysed cells. Cytoplasmic protein was extracted from centrifuged supernatant (at $14,000 \mathrm{~g}$ for $30 \mathrm{~min}$ at $4^{\circ} \mathrm{C}$ ). The total membrane pellet was resuspended in the upper phase solution with $1 \mathrm{mM}$ PMSF; then the lower phase solution was added, incubated on ice for $5 \mathrm{~min}$, and centrifuged at $1000 \times \mathrm{g}$ for $5 \mathrm{~min}$ at $4^{\circ} \mathrm{C}$. The upper phase solution was carefully collected and then centrifuged at $15,000 \times \mathrm{g}$ for $10 \mathrm{~min}$ at $4^{\circ} \mathrm{C}$. Remove the supernatant; the pellet was the PM protein and can be dissolved in RIPA before further use. All proteins were then quantitated by BCA protein assay (Beyotime Institute of Biotechnology, Shanghai, China) and were stored at $-80^{\circ} \mathrm{C}$. All proteins were analyzed by immunoblotting.

2.7. Western Blot (WB). $20 \mu \mathrm{g}$ protein were separated by $8.0 \%$ SDS-polyacrylamide gel electrophoresis and transferred to a polyvinylidene fluoride (PVDF) membrane. After transfer, the PVDF membranes were blocked with 5\% (w/v) skim milk

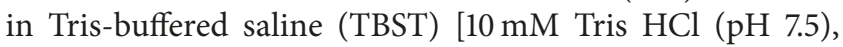
$150 \mathrm{mM} \mathrm{NaCl}$, and $0.1 \%$ Tween-20] for $1 \mathrm{~h}$ at room temperature (RT). The PVDF membranes were then blotted with rabbit polyclonal PKC $\gamma$ antibody (1:2000, Abcam Co., Ltd., USA) or rabbit monoclonal PKCe antibody (1:1000, Santa Cruz, USA), or rabbit TRPV1 polyclonal antibody $(1: 1000$, Abcam Co., Ltd., USA), or rabbit p-TRPV1 polyclonal antibody (1:1000, Abcam Co., Ltd., USA), or rabbit monoclonal GAPDH antibody (1:10000, Abcam Co., Ltd., USA), or rabbit polyclonal $\mathrm{Na}^{+}-\mathrm{K}^{+}$-ATPase antibody $(1: 1000$, Cell Signaling Technology, USA) for the DRG and SCDH samples at $4^{\circ} \mathrm{C}$ overnight in 5\% skim milk. Then, the samples were incubated with HRP conjugated goat anti-rabbit antibody for $2 \mathrm{~h}$ at RT, the bands were detected by chemiluminescence using
Western ECL kit (Abcam Co., Ltd., USA), and densitometry was performed by Image Quant LAS 4000 (GE, USA). The integrated optical density (IOD) of the bands was analyzed by Image Quant TL7.0 Analysis Software (GE, USA).

2.8. Immunofluorescence Assay. The rats were deeply anesthetized intraperitoneally with sodium chloral hydrate $(0.35 \mathrm{~mL} / 100 \mathrm{~g})$ and then perfused with $4 \%$ paraformaldehyde in $0.1 \mathrm{M}$ phosphate buffer $\left(\mathrm{pH} 7.4,4^{\circ} \mathrm{C}\right) . \mathrm{SCDH}$ and DRG were fixed in $4 \%$ paraformaldehyde for $4 \mathrm{~h}$, transferred into $15 \%$ sucrose overnight and then $30 \%$ sucrose for $48 \mathrm{~h}$ in $0.1 \mathrm{M}$ phosphate buffer ( $\mathrm{pH} 7.4)$ for cryoprotection, and stored at $-80^{\circ} \mathrm{C}$. DRG at $12 \mathrm{~mm}$ and $\mathrm{SCDH}$ at $30 \mathrm{~mm}$ were cut by freezing microtome (HM550, Thermo Fisher Scientific, USA) and mounted on slides. To permit comparisons across treatment groups, sections from different groups were processed simultaneously. The slides were incubated in $10 \%$ $(\mathrm{w} / \mathrm{v})$ normal goat serum for 1 hour and then incubated overnight at $4^{\circ} \mathrm{C}$ in rabbit polyclonal TRPV1 antibody (DRG, 1:1000; SCDH, 1:400, Abcam Co., Ltd., USA) or rabbit polyclonal PKCe antibody (1:500, Abcam Co., Ltd., USA) or rabbit monoclonal PKC $\gamma$ (1:500, Abcam Co., Ltd., USA). After washing, slides were transferred to anti-goat IgG H\&L (Alexa Fluor @645) (TRPV1, 1:400; PKCe, 1:200, Abcam Co., Ltd., USA) or anti-rabbit IgG H\&L (Alexa Fluor @488) (PKC $\gamma, 1: 200$, Abcam Co., Ltd., USA) for 1 hour at $37^{\circ} \mathrm{C}$. Sections were mounted on glass slides, air-dried, and covered with coverslips by Aquamount (Thermo Fisher Scientific, USA). Images were captured at $100 \mathrm{x}$ magnification by a laser scanning confocal microscope (Nikon A1R/A1, Japan). Quantification of immunoreactivity (IR) was performed by calculating the number of TRPV1-IR neurons in the total number of neurons in the DRG and the total optical density of the positive neuron in laminae I-II of the SCDH in each section using Image-Pro Plus 6.0 software. Five sections per animal were randomly selected, and the mean ratio was determined. All DRG neurons were sectioned through their nuclei.

2.9. Statistical Analysis. All data are expressed as the mean \pm SEM. Statistically significant differences among groups were determined by one-way analysis of variance (ANOVA). The differences for multiple comparisons were performed by least significant difference (LSD) testing (if there was variance homogeneity) and Dunnett's T3 test (if there was variance heterogeneity). $P<0.05$ was considered statistically significant in this study.

\section{Results}

3.1. Intrathecal Administration of MrgprC siRNA Blocks Analgesic Effect of EA. The ipsilateral PWL of rats was used to assess thermal hyperalgesia (Figure 1). Before CFA injection, there were no differences among groups. Compared with the normal group, PWLs in the group with CFA injection showed a drastic decrease on day 1 after CFA injection $(P<0.01)$. Compared with the model group, the PWLs of rats given EA or BAM8-22 increased gradually at each time point from day 3 or day $2(P<0.01, P<0.01$, resp.). There was a significant 


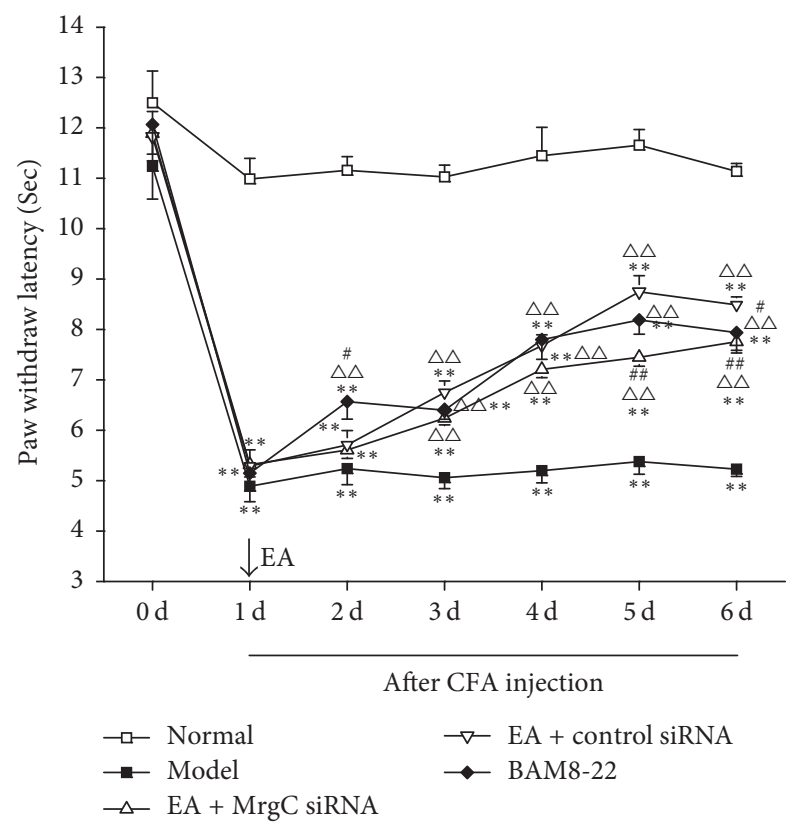

FIGURE 1: Behavioral test at different time point. Ipsilateral PWLs at different time points were assessed by plantar test apparatus. Data are expressed as the mean $\pm \operatorname{SEM}(n=8) .{ }^{* *} P<0.01$ compared to normal group, ${ }^{\triangle \triangle} P<0.01$, compared to model group, and ${ }^{\# \#} P<$ 0.01 and ${ }^{\#} P<0.05$ compared to EA + control siRNA group.

reduction in PWL in the EA + MrgprC siRNA group compared with the EA + control siRNA group when MrgprC siRNA was intrathecally administered on day 5 and day $6(P<0.01$, $P<0.01$, resp.).

3.2. Regulation of EA on $p-T R P V 1$ and TRPV1 Translocation, but Not TRPVI Expression, Is Inhibited by MrgprC siRNA in the DRG. To investigate the influence of MrgprC on EA modulation of the TRPV1 pathway in the DRG, we observed the expression of TRPV1, TRPV1 translocation, and $\mathrm{p}$ TRPV1. Compared with the normal group, TRPV1 expression in the DRG by WB markedly increased on the 6th day after CFA injection $(P<0.01)$. Six doses of EA downregulated the expression of TRPV1 compared with the model group $(P<0.05)$. However, intrathecal MrgprC siRNA had no effect on TRPV1 expression in the EA + MrgprC siRNA group compared with the EA + control siRNA group. Intrathecal BAM822 also significantly downregulated expression of TRPV1. Immunofluorescence was also performed to detect TRPV1 expression. Positive TRPV1-IR was highly distributed in small neurons. In line with data from WB (Figure 2(a)), immunofluorescence (Figure 2(b)) data showed CFA injection increased TRPV1 expression in the model group $(P<0.01)$. The increase was attenuated by EA $(P<0.05)$ or intrathecal BAM8-22 $(P<0.01)$. Intrathecal MrgprC siRNA did not inhibit the downregulation of TRPV1 by EA.

To explore the influence of MrgprC siRNA on TRPV1 translocation, the expression of TRPV1 in the PM and the cytoplasm was detected (Figure 3). The translocation of
TRPV1 obviously increased in the model group when compared with the normal group $(P<0.01)$. EA and intrathecal BAM8-22 both resulted in a decrease in translocation $(P<$ $0.01, P<0.01)$. Compared with EA + control siRNA group, intrathecal MrgprC siRNA can effectively suppress EA modulation on the translocation of TRPV1 $(P<0.01)$.

Then, we observed the alteration of p-TRPV1 levels in the DRG (Figure 4). Levels of p-TRPV1 in the model group were higher than in the normal group $(P<0.01)$. EA downregulated p-TRPV1 levels in EA + control siRNA groups compared with the model group $(P<0.01)$. Intrathecal MrgprC siRNA obviously inhibited downregulation of EA on p-TRPV1 $(P<0.01)$. Compared with the model group, $\mathrm{p}$-TRPV1 levels in the BAM8-22 group were significantly reduced in DRG $(P<0.01)$.

\subsection{Regulation of EA on $p-T R P V 1$ and TRPV1 Translocation,} but Not TRPV1 Expression, Is Inhibited by MrgprC siRNA in the SCDH. We then explored the influence of MrgprC siRNA on expression of TRPV1, TRPV1 translocation, and p-TRPV1 in the SCDH through $\mathrm{WB}$ and immunofluorescence. The TRPV1 expression obviously increased in the model group when compared with the normal group $(P<0.01)$. EA and intrathecal BAM8-22 both resulted in a decrease in TRPV1 expression $(P<0.01, P<0.01)$. Nevertheless, expression in the EA + MrgprC siRNA group was not significantly higher than that in the EA + control siRNA group when intrathecally administered with MrgprC siRNA (Figures 5(a) and 5(b)).

The influence of MrgprC siRNA on the translocation of TRPV1 was next investigated (Figure 6). The translocation obviously increased in the model group when compared with the normal group $(P<0.01)$. EA and intrathecal BAM8-22 both resulted in a decrease in translocation $(P<0.05, P<$ $0.05)$. Translocation in the EA + MrgprC siRNA group was higher than in the EA + control siRNA group when intrathecally administered with MrgprC siRNA $(P<0.05)$.

We also observed an alteration of p-TRPV1 levels in the SCDH (Figure 7). p-TRPV1 levels in the model group were higher than those in the normal group in the SCDH $(P<$ 0.01). EA downregulated $\mathrm{p}$-TRPV1 in the EA + control siRNA group compared with the model group $(P<0.01)$. However, intrathecal MrgprC siRNA obviously inhibited the effect of EA on p-TRPV1 $(P<0.05)$. Compared with the model group, the p-TRPV1 levels in the BAM8-22 group were significantly reduced $(P<0.01)$.

3.4. MrgprC siRNA Blocks Downregulation Effect of EA on $P K C \varepsilon$ in the DRG but Not on PKC $\gamma$ in the SCDH. We next investigated whether MrgprC modulated the EA intervention through PKC in the DRG and SCDH as it is linked closely to the phosphorylation of TRPV1 [26]. PKC $\varepsilon$ is one of the key subtypes of PKC highly expressed in the DRG, and PKC $\gamma$ is highly expressed in the SCDH. WB and immunofluorescence were performed to investigate these expressions (Figures 8 and 9). The data from WB showed that the CFA injection produced an increase in PKCe $(P<0.05)$ and PKC $\gamma(P<$ 0.01 ) in the model group compared to the normal group on the inflamed side. The increase was significantly inhibited by EA (PKC $\varepsilon, P<0.01$; PKC $\gamma, P<0.01)$. When MrgprC 


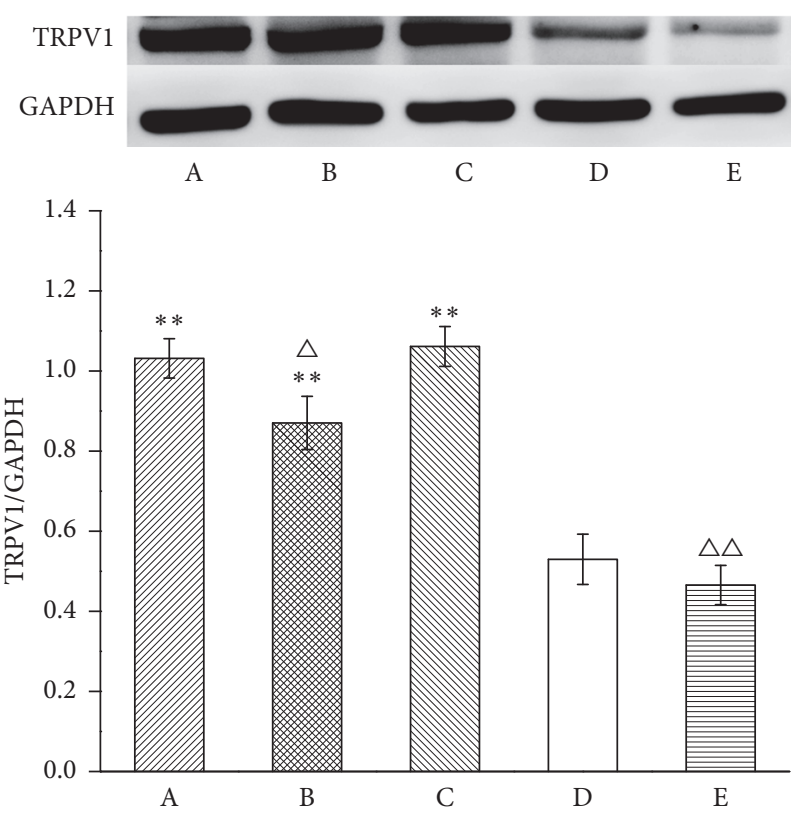

(a)
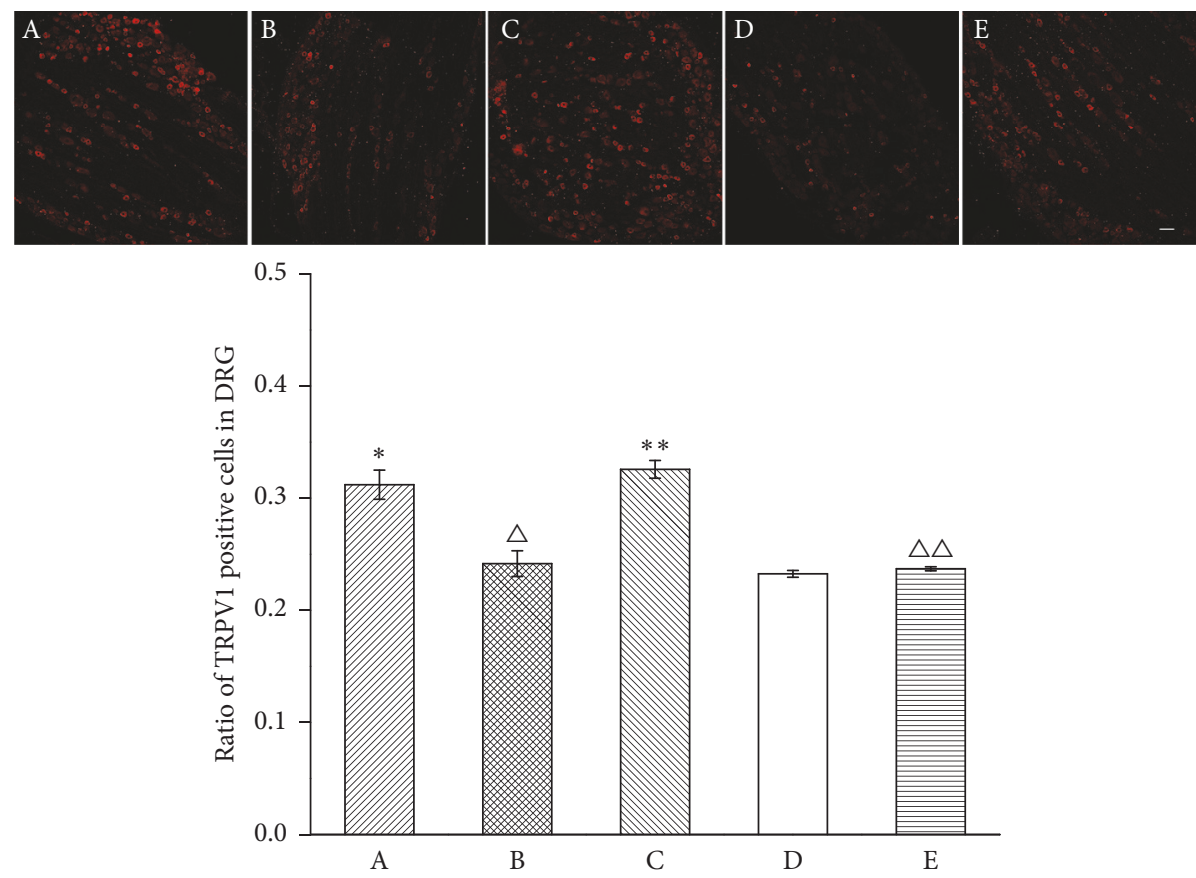

(b)

FIGURE 2: TRPV1 expression in ipsilateral DRG. (a) Results from WB are expressed as relative density of TRPV1 (GAPDH serves as the commonly used internal reference protein) $(n=4)$. (b) TRPV1 positive neurons were mainly expressed in small-to-medium DRG cells. The results from immunofluorescence are expressed as proportion of TRPV1-IR positive cells over whole stained cells $(n=4)$. Scale bar $=100 \mu \mathrm{m}$. Data are expressed as the mean \pm SEM. ${ }^{*} P<0.01$ and ${ }^{* *} P<0.01$ compared to normal group; ${ }^{\triangle} P<0.05$ and ${ }^{\triangle \triangle} P<0.01$ compared to model group. A: EA + MrgprC siRNA group, B: EA + control siRNA group, C: model group, D: normal group, and E: BAM8-22 group.

siRNA was intrathecally administered, expression of PKCE was significantly higher in the EA + MrgprC siRNA group than in the EA + control siRNA group, but there was no difference in PKC $\gamma$ levels between them. Meanwhile, intrathecal BAM8-22 attenuated CFA-induced increase in PKC $\varepsilon$ $(P<0.05)$ in the DRG and PKC $\gamma$ in the SCDH $(P<0.01)$.
Immunofluorescence was also used to observe the expression of PKC. The data from immunofluorescence were consistent with the data from WB. Figures 8(b) and 9(b) showed that there were basal levels of PKC $\varepsilon$ in the DRG and PKC $\gamma$ in the SCDH. Compared with the normal group, CFA injection caused a robust increase in PKCE $(P<0.01)$ in the DRG and 


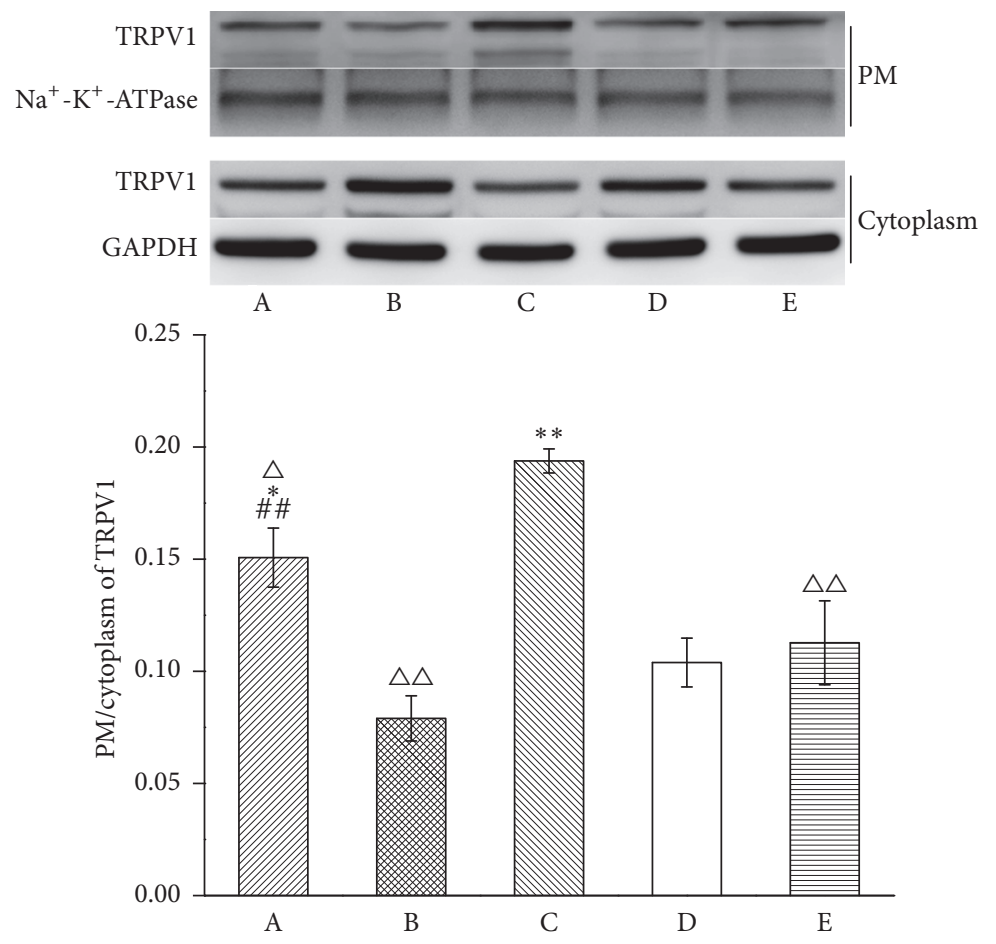

FIgURE 3: Translocation of TRPV1 in ipsilateral DRG. The results from WB are expressed as relative density of TRPV1 in the PM $\left(\mathrm{Na}^{+}-\mathrm{K}^{+}-\right.$ ATPase serves as the commonly used internal reference protein in the PM and GAPDH serves as the commonly used internal reference protein in the cytoplasm) $(n=4)$. Data are expressed as the mean \pm SEM. ${ }^{*} P<0.05$ and ${ }^{* *} P<0.01$ compared to normal group; ${ }^{\triangle} P<0.05$ and ${ }^{\triangle \triangle} P<0.01$ compared to model group; ${ }^{\# \#} P<0.01$ compared to EA + control siRNA group. A: EA + MrgprC siRNA group, B: EA + control siRNA group, C: model group, D: normal group, and E: BAM8-22 group.

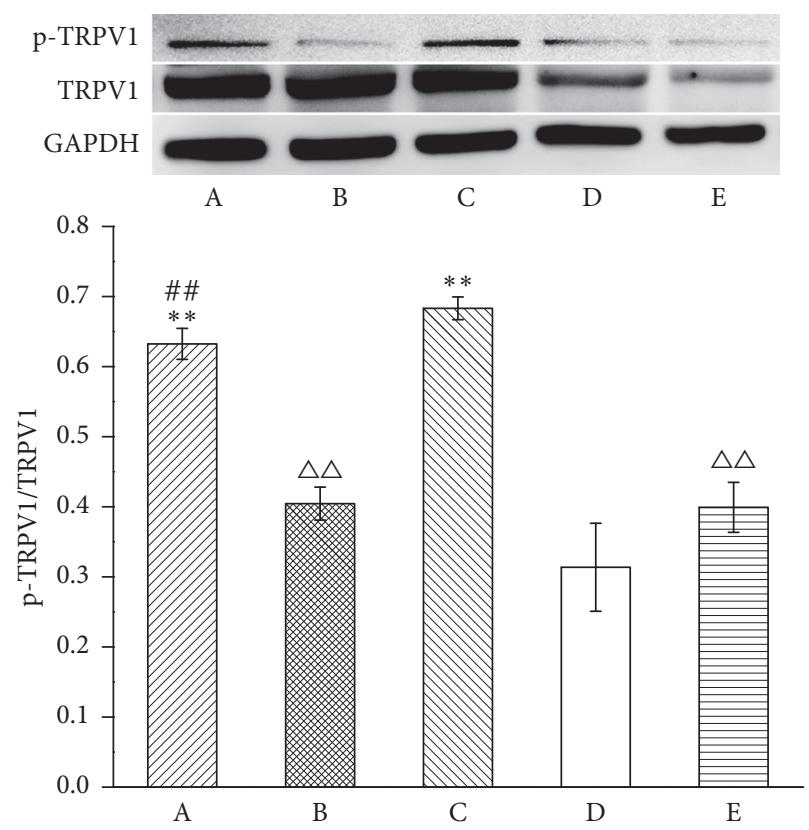

FIGURE 4: p-TRPV1 level in ipsilateral DRG. Results from WB are expressed as relative density of p-TRPV1 (equal loading was verified by assaying TRPV1) $(n=4)$. Data are expressed as the mean \pm SEM. ${ }^{* *} P<0.01$ compared to normal group; ${ }^{\triangle \Delta} P<0.01$ compared to model group; ${ }^{\# \#} P<0.01$ compared to EA + control siRNA group. A: EA + MrgprC siRNA group, B: EA + control siRNA group, C: model group, D: normal group, and E: BAM8-22 group. 


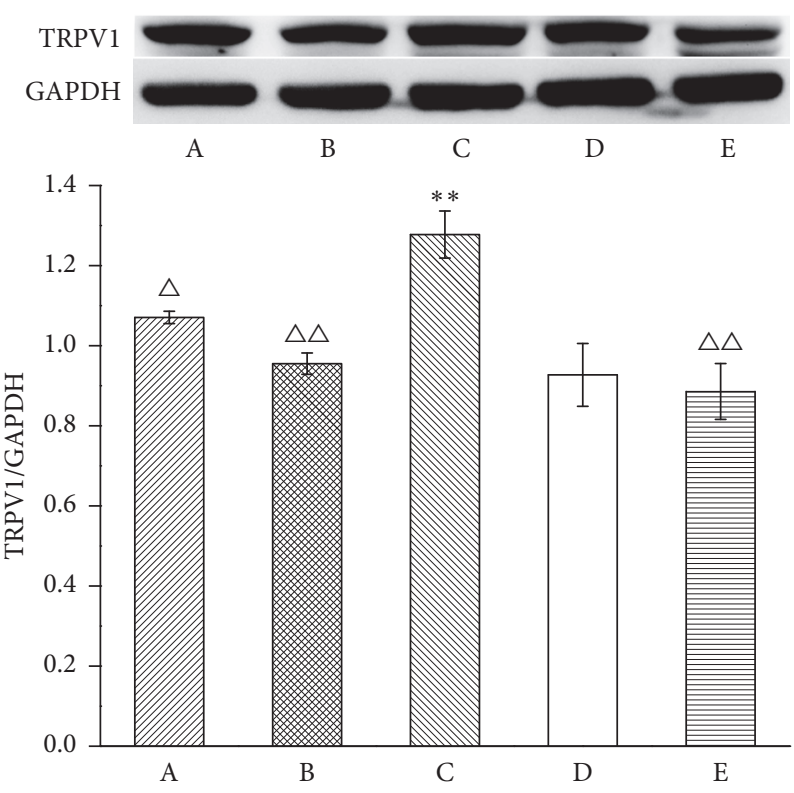

(a)
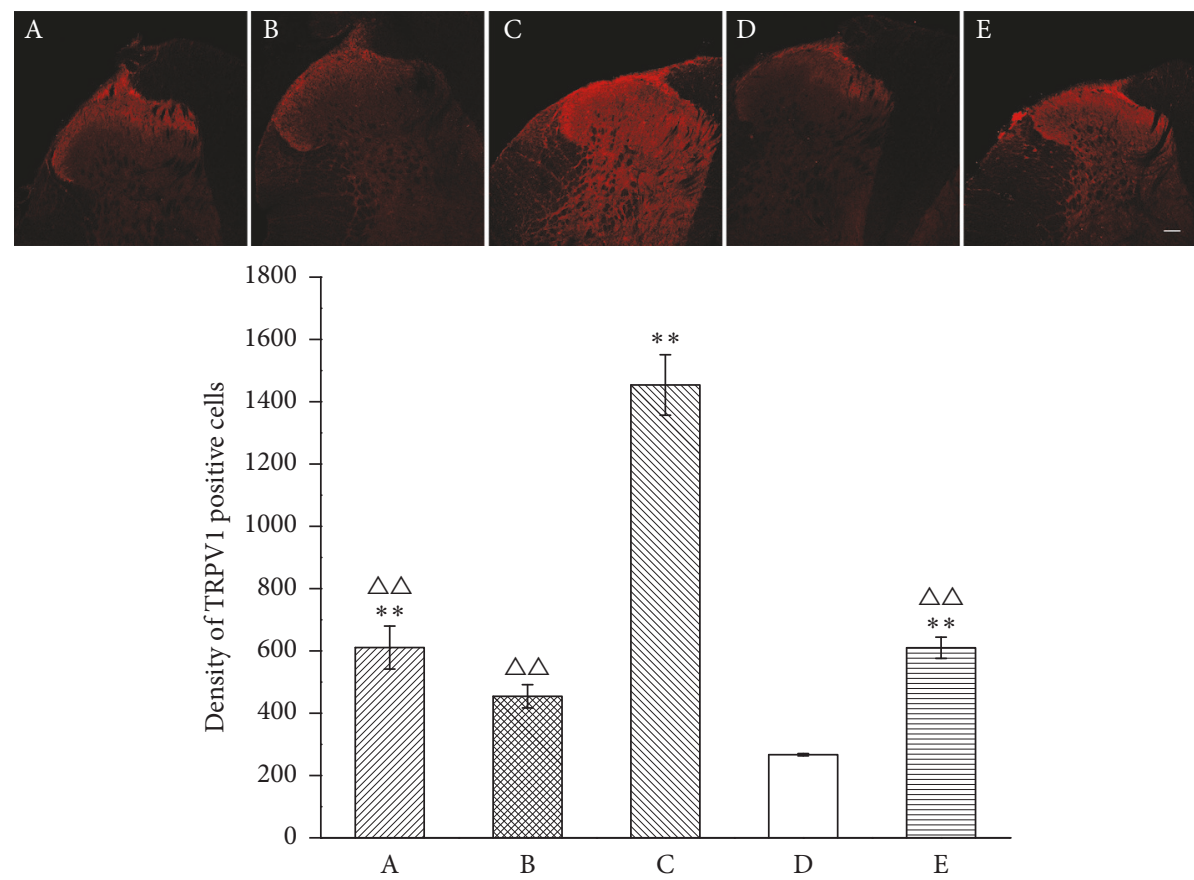

(b)

FIgURE 5: TRPV1 expression in ipsilateral SCDH. (a) Results from WB are expressed as relative density of TRPV1 (GAPDH serves as the commonly used internal reference protein) $(n=4)$. (b) TRPV1 positive neurons were mainly expressed in superficial layer of SCDH (laminae I-II). The results from immunofluorescence are expressed as total density of TRPV1-IR positive cells in laminae I-II $(n=4)$. Scale bar $=$ $100 \mu \mathrm{m}$. Data are expressed as the mean \pm SEM. ${ }^{* *} P<0.01$ compared to normal group; ${ }^{\triangle} P<0.05$ and ${ }^{\triangle \triangle} P<0.01$ compared to model group. A: EA + MrgprC siRNA group, B: EA + control siRNA group, C: model group, D: normal group, and E: BAM8-22 group.

PKC $\gamma(P<0.01)$ in the SCDH. EA downregulated the expression of $\mathrm{PKC} \varepsilon(P<0.01)$ and $\mathrm{PKC} \gamma(P<0.01)$ compared with the normal group. When MrgprC siRNA was intrathecally administered, the regulation effect of EA was blocked in the DRG but not in the SCDH. In addition, the increase in PKC $\varepsilon(P<0.01)$ and PKC $\gamma(P<0.05)$ induced by CFA was also inhibited by BAM8-22.

\section{Discussion}

Peripheral sensitization includes thermal hyperalgesia and mechanical allodynia, which are the main features of chronic inflammatory pain. Ipsilateral hyperalgesia is generated within $4 \mathrm{~h}$ after CFA modeling and may last for 4-6 weeks, while contralateral hyperalgesia generally occurs 18 days 


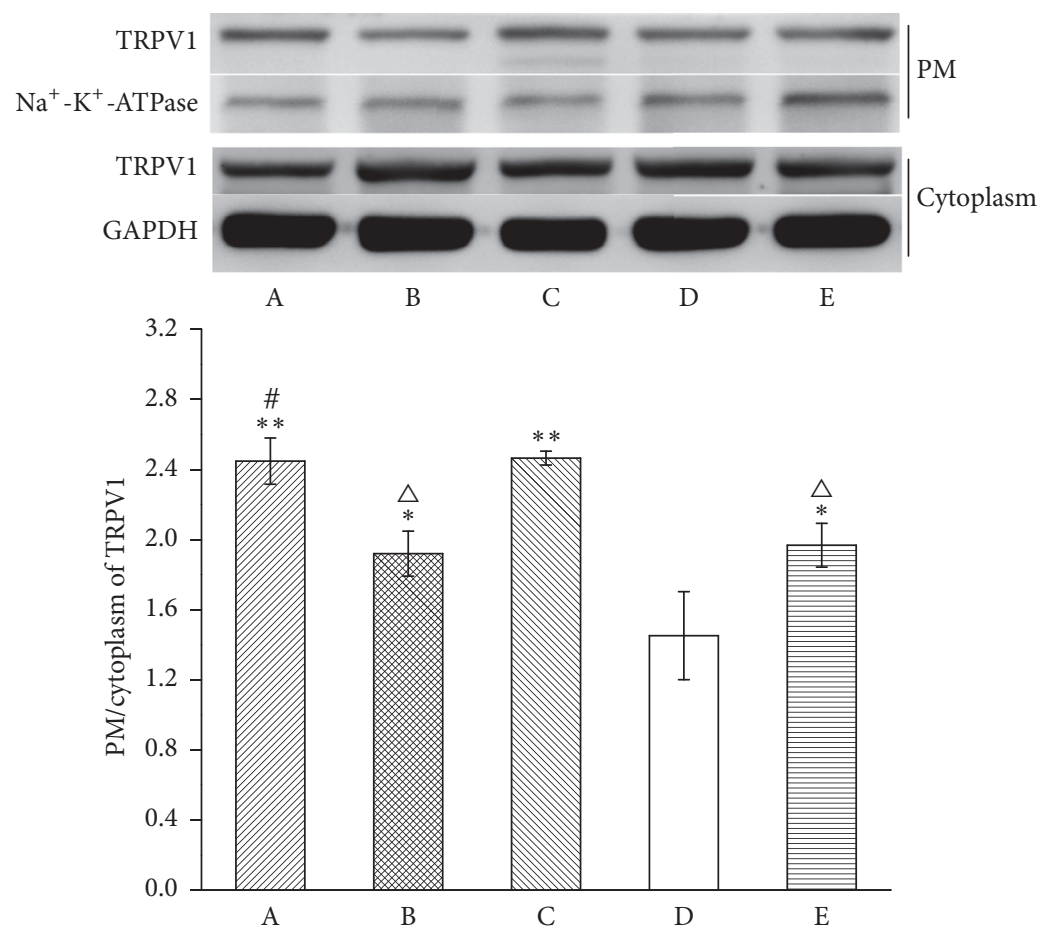

FIGURE 6: Translocation of TRPV1 in ipsilateral SCDH. The results from WB are expressed as relative density of TRPV1 in the PM (Na ${ }^{+}-$ $\mathrm{K}^{+}$-ATPase serves as the commonly used internal reference protein in the PM and GAPDH serves as the commonly used internal reference protein in the cytoplasm) $(n=4)$. Data are expressed as the mean \pm SEM. ${ }^{*} P<0.05$ and ${ }^{* *} P<0.01$ compared to normal group; ${ }^{\triangle} P<0.05$ compared to model group; ${ }^{\#} P<0.05$, compared to EA + control siRNA group. A: EA + MrgprC siRNA group, B: EA + control siRNA group, C: model group, D: normal group, and E: BAM8-22 group.

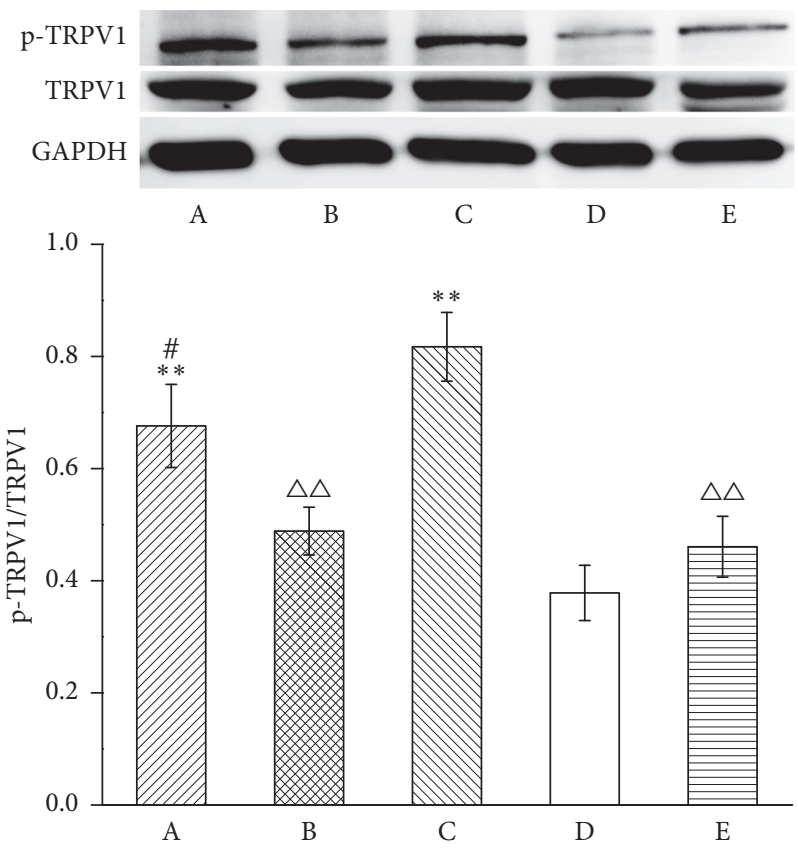

FIGURE 7: p-TRPV1 level in ipsilateral SCDH. Results from WB are expressed as relative density of p-TRPV1 (equal loading was verified by assaying TRPV1) $(n=4)$. Data are expressed as the mean \pm SEM. ${ }^{* *} P<0.01$ compared to normal group; ${ }^{\triangle \Delta} P<0.01$ compared to model group; ${ }^{\#} P<0.05$ compared to EA + control siRNA group. A: EA + MrgprC siRNA group, B: EA + control siRNA group, C: model group, D: normal group, and E: BAM8-22 group. 


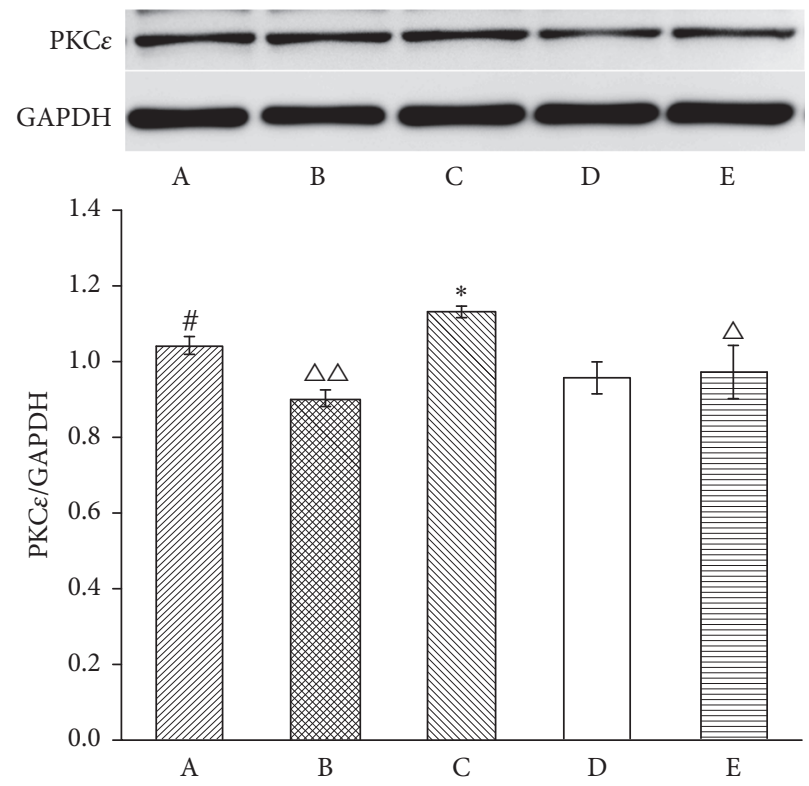

(a)
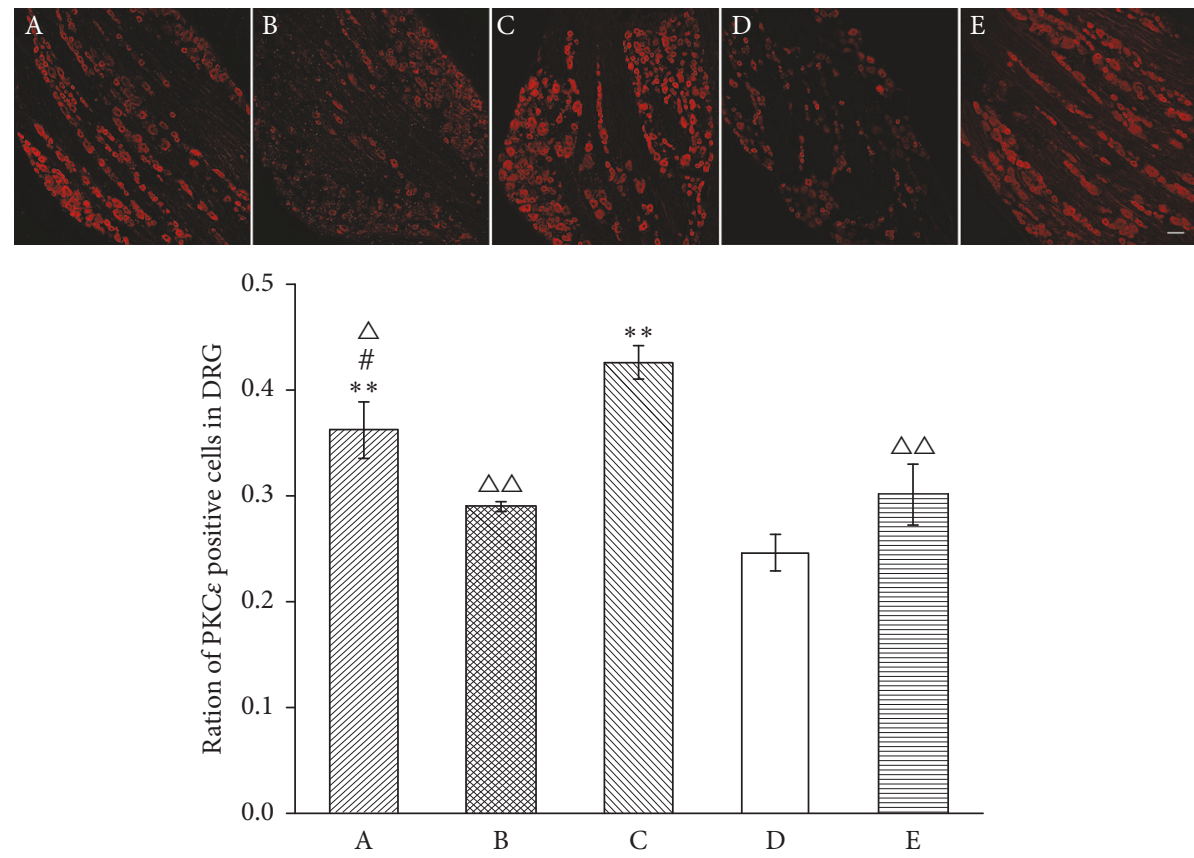

(b)

FIgURE 8: Expression of PKCe in ipsilateral DRG. (a) Results from WB are expressed as relative density of PKCe (GAPDH serves as the commonly used internal reference protein) $(n=4)$. (b) PKC $\varepsilon$-IR positive neurons were mainly expressed in small-to-medium DRG cells. The results from immunofluorescence are expressed as ratio of PKCe-IR positive cells over whole stained cells $(n=4)$. Scale bar $=100 \mu \mathrm{m}$. Data are expressed as the mean \pm SEM. ${ }^{*} P<0.05$ and ${ }^{* *} P<0.01$, compared to normal group; ${ }^{\triangle} P<0.05$ and ${ }^{\triangle \triangle} P<0.01$ compared to model group; ${ }^{\#} P<0.05$ compared to EA + control siRNA group. A: EA + MrgprC siRNA group, B: EA + control siRNA group, C: model group, D: normal group, and E: BAM8-22 group.

after CFA modeling [27]. Our previous study showed that there was no effect on mechanical allodynia with intrathecal administration of MrgprC siRNA that was sufficient for blocking MrgprC expression in the DRG [28]. Therefore, ipsilateral PWL was observed to assess thermal hyperalgesia in this study. After subcutaneously injecting with CFA for
1 day, the ipsilateral hind paws of rats showed redness, swelling, and heat. The PWL of the model group was significantly lower than that of the saline group from $1 \mathrm{~d}$ to $6 \mathrm{~d}$, which suggested that the model was successfully established. Previous studies demonstrated that $2 / 100 \mathrm{~Hz}$ EA stimulating bilateral ST36 and the BL60 can alleviate thermal 


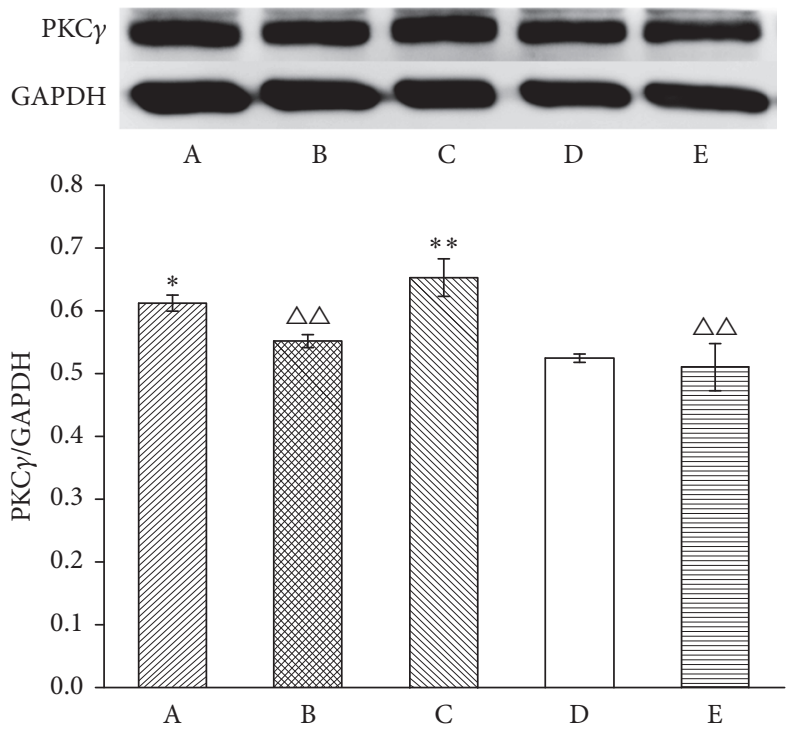

(a)
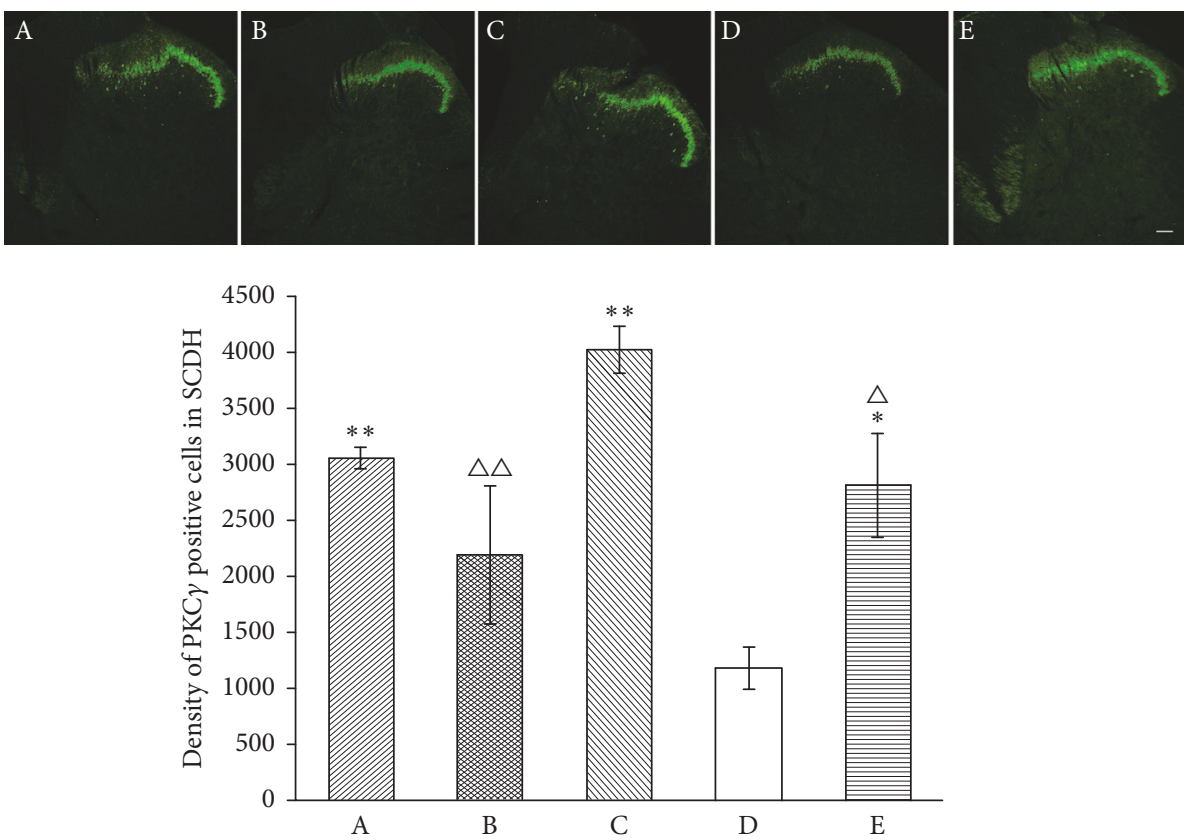

(b)

FIGURE 9: Expression of PKC $\gamma$ in ipsilateral SCDH. (a) Results from WB are expressed as relative density of PKC $\gamma$ (GAPDH serves as the commonly used internal reference protein) $(n=4)$. (b) PKC $\gamma$-IR positive neurons were mainly expressed in lamina II of superficial spinal dorsal horn. The results from immunofluorescence are expressed as density of PKC $\gamma$-IR positive cells in lamina II $(n=4)$. Scale bar $=100 \mu \mathrm{m}$. Data are expressed as the mean \pm SEM. ${ }^{*} P<0.05$ and ${ }^{* *} P<0.01$ compared to normal group; ${ }^{\triangle} P<0.05$ and ${ }^{\triangle \triangle} P<0.01$ compared to model group. A: EA + MrgprC siRNA group, B: EA + control siRNA group, C: model group, D: normal group, and E: BAM8-22 group.

hyperalgesia induced by chronic inflammation $[8,16,23]$, especially on the 5th and 9th day after CFA modeling [29]. We observed 6 instances of EA alleviating thermal hyperalgesia in this study.

MrgprC is one of the members of the superfamily associated with CGRPs, and its gene is highly expressed in the pain-related peripheral nervous system (DRG, trigeminal ganglion) that is closely related to pain transmission. The Mrg receptor family is classified as MrgprA-MrgprH in rodents [11] and MrgprX1-MrgprX7 in humans [30]. A latest research shows MRGPRX is a promising therapeutic target for treating persistent pain because of its unique expression in nociceptors within the peripheral nervous system [31]. Studies have shown that rodent MrgprC and human MrgprX are 
structurally highly homologous [32]. Therefore, elucidating the role of MrgprC in pain can contribute to a better understanding of the role of human MrgprX in pain, since there was no direct evidence that MrgprC had a modulatory effect on chronic inflammatory pain as an MrgprC-specific inhibitor had not been found yet. The current studies are limited to investigating the effect of different MrgprC agonists on pain and effects of MrgprC in the early stage of CFA models (24-48 h) $[33,34]$. These studies showed that intrathecal discontinuous injection of BAM8-22 could suppress thermal hyperalgesia in chronic inflammatory pain. Consistent with other papers, our early research showed that intrathecal injection of MrgprC siRNA more than 5 times can block 50-60\% of MrgprC gene expression [14, 28, 35]. To elucidate the influence of MrgprC on EA analgesia, MrgprC siRNA was intrathecally injected 6 times with mismatched siRNA as a control. The results showed that the analgesic effect of EA decreased after applying MrgprC siRNA. Considering our previous data $[16,36]$ showed that MrgprC expression in the DRG but not in the SCDH and BAM22 release in both the DRG and the SCDH could be increased by EA in CFAinduced chronic inflammatory pain, the results indicated that MrgprC may play a role in modulation of EA over a 6day period of chronic inflammatory pain, and the possible analgesic mechanism of EA is associated with its upregulation on MrgprC in the DRG and activation of MrgprC by BAM22 in both the DRG and SCDH.

Persistent inflammatory injury can trigger and maintain nociceptive thermal hyperalgesia, and TRPV1 is usually considered to play a key role in inflammatory and thermal pain [37]. TRPV1 is highly expressed in neurons in the DRG [38], and deletion of TRPV1 results in thermal hyperalgesia elicited by CFA [39]. Consistent with previous research, TRPV1 expression was significantly increased in the DRG and SCDH 6 days after CFA modeling in this study. The results of the immunofluorescence assay indicated that the TRPV1 increase was mainly in DRG small neurons and superficial layer of the SCDH. In the 28-day chronic pain caused by CFA, TRPV1 in isolectin B4 (IB4) positive (signal of MrgprC positive DRG neurons [40]) DRG neurons was consistently overexpressed in the first 14 days and further transported into the superficial layer of the SCDH [41]. TRPV1 was shown to be a major downstream target in the MrgprC-induced cell signaling pathway in acute inflammatory pain, and the effect of MrgprC on TRPV1 was mediated by PKC [21]. It is presumed that MrgprC may suppress CFA-induced hyperalgesia at the DRG and SCDH levels based on these data. Upon expression, a part of MrgprC is transported to the terminal of primary afferent synapses in superficial layer II of the SCDH that is bound by PKC $\gamma$ [42]. MrgprC activated by BAM22 can downregulate the expression of CGRP in the L3-L5 layer of the SCDH and DRG and inhibit occurrence and development of inflammatory hyperalgesia induced by CFA in the early stage $(24 \mathrm{~h})$ [43]. Intrathecal injection of BAM8-22 attenuated CFA-induced thermal hyperalgesia and inhibited ERK phosphorylation in the DRG and at the cytomembrane PKC $\gamma$ level in the SCDH [3]. Thus, we observed how MrgprC takes part in EA analgesia through TRPV1 pathways in the DRG and SCDH. The present results showed EA and BAM8-22 effectively inhibited TRPV1 expression, whereas MrgprC did not participate in EA inhibition of TRPV1 expression in the DRG and SCDH.

At the subcellular level, TRPV1 mostly seems to be a kind of sequestered intracellular compartments [44]. In the process of inflammatory pain, TRPV1 can be activated by SP, calcitonin peptide, and others [45]; then, it translocates to the $\mathrm{PM}$ from the cytoplasm and is phosphorylated by PKC/PKA and finally the function of TRPV1 is activated [46]. In this study, we observed the expression of TRPV1 in the PM and cytoplasm to investigate the translocation of TRPV1 in the DRG and SCDH. The results showed that the translocation of TRPV1 was increased in chronic inflammatory pain and the suppression of EA could be reversed by MrgprC siRNA. It has been confirmed that MrgprC-contained primary afferent fibers terminate in the middle region of the SCDH that is rich in PKC $\gamma$ subtype neurons [33, 40, 47]. Therefore, the function of MrgprC might be closely related to PKC $\gamma$. A previous study showed that inhibition of the phosphorylation of PKC $\varepsilon$ at the ser729 site in DRG promoted by MrgprC could inhibit the functional activation of PKCE [28]. In this experiment, it was observed that both EA and BAM8-22 could inhibit the upregulation of inflammation on expression of PKC and PKC-mediated phosphorylation of TRPV1 in the DRG and SCDH. Furthermore, MrgprC siRNA reversed the inhibition of EA on the expression of PKC $\varepsilon$ and p-TRPV1 but did not inhibit the expression of PKC $\gamma$ in the SCDH, which suggested other potential ways for EA modulation on MrgprC to decrease the phosphorylation of TRPV1 in the SCDH. MrgprC was involved in the regulation of EA on the translocation and the phosphorylation of TRPV1 via PKC in different ways in the DRG and SCDH.

In this study, it was observed that PKCe in the DRG, $\mathrm{PKC} \gamma$ in the SCDH, the translocation, and phosphorylation of TRPV1 in the DRG and SCDH were significantly increased after 6 days of CFA modeling, the upregulation of which was inhibited by BAM8-22. Thus, MrgprC may be involved in the modulation of chronic inflammatory pain through the PKC/TRPV1 pathway in the DRG and SCDH. EA also inhibited the translocation and phosphorylation of TRPV1 as well as the expression of PKCE in the DRG and PKC $\gamma$ in the SCDH. MrgprC siRNA reversed the effect of EA on PKCe expression, the translocation, and the phosphorylation of TRPV1 but did not reverse PKC $\gamma$ expression, which suggested another potential way for EA modulation on MrgprC to decrease the phosphorylation of TRPV1 in the SCDH. All the above data showed that MrgprC was involved in EA regulation of the PKC/TRPV1 pathway in different ways in the DRG and SCDH.

\section{Conclusions}

In summary, this current study shows a novel finding that EA and MrgprC both play a role in chronic inflammatory pain regulation by inhibiting expression, translocation, and PKC-mediated phosphorylation of TRPV1 in the DRG and SCDH. MrgprC may be involved in EA analgesia through the PKC/TRPV1 pathway in the DRG and SCDH in different ways. The above results demonstrate the functional role of EA 
in pain signaling and provide a novel peripheral mechanism for involvement of MrgprC, an atypical opioid receptor, in EA regulation of chronic inflammatory pain.

\section{Conflicts of Interest}

The authors have no conflicts of interest to declare.

\section{Authors' Contributions}

Fang Fang and Jian-qiao Fang contributed equally to this work.

\section{Acknowledgments}

This study was supported by grants from the National Natural Science Foundation of China (no. 81202755), the Zhejiang Provincial Natural Science Foundation of China (no. LY17H270014), and the Projects of Medical and Health Technology Program in Zhejiang Province (no. 2015KYA172).

\section{References}

[1] T. Jackson, S. Thomas, V. Stabile, X. Han, M. Shotwell, and K. McQueen, "Prevalence of chronic pain in low-income and middle-income countries: a systematic review and meta-analysis," The Lancet, vol. 385, p. S10, 2015.

[2] R. Zhang, L. Lao, K. Ren, and B. M. Berman, "Mechanisms of acupuncture-electroacupuncture on persistent pain," Anesthesiology, vol. 120, no. 2, pp. 482-503, 2014.

[3] D. Wang, P. Wang, J. Jiang, Q. Lv, X. Zeng, and Y. Hong, "Activation of mas oncogene-related g protein-coupled receptors inhibits neurochemical alterations in the spinal dorsal horn and dorsal root ganglia associated with inflammatory pain in rats," The Journal of Pharmacology and Experimental Therapeutics, vol. 354, no. 3, pp. 431-439, 2015.

[4] R. R. Ji, Q. Zhang, P. Y. Law, and et al., "Expression of mu-, delta-, and kappa-opioid receptor-like immunoreactivities in rat dorsal root ganglia after carrageenan-induced inflammation," Journal of Neuroscience, vol. 15, no. 12, pp. 8156-8166, 1995.

[5] J. Han, "Acupuncture analgesia: areas of consensus and controversy," PAIN, vol. 152, no. 3, pp. S41-S48, 2011.

[6] T. Matsubara, Y.-C. P. Arai, Y. Shiro et al., "Comparative effects of acupressure at local and distal acupuncture points on pain conditions and autonomic function in females with chronic neck pain," Evidence-Based Complementary and Alternative Medicine, vol. 2011, Article ID 543291, 6 pages, 2011.

[7] J. R. Peterson, "Acupuncture in the 1990s: A Review for the Primary Care Physician," Archives of Family Medicine, vol. 5, no. 4, pp. 237-240, 1996.

[8] J.-Q. Fang, J.-Y. Du, Y. Liang, and J.-F. Fang, "Intervention of electroacupuncture on spinal p38 MAPK/ATF-2/VR-1 pathway in treating inflammatory pain induced by CFA in rats," Molecular Pain, vol. 9, no. 1, article 13, 2013.

[9] J.-Q. Fang, Y. L. Jiang, S. C. Qiu et al., "Involvement of peripheral beta-endorphin and mu, delta, kappa opioid receptors in electroacupuncture analgesia for prolonged inflammatory pain of rats," European Journal of Inflammation, vol. 11, no. 2, pp. 375383, 2013

[10] E. Grazzini, C. Puma, M.-O. Roy et al., "Sensory neuron-specific receptor activation elicits central and peripheral nociceptive effects in rats," Proceedings of the National Acadamy of Sciences of the United States of America, vol. 101, no. 18, pp. 7175-7180, 2004.

[11] X. Dong, S.-K. Han, M. J. Zylka, M. I. Simon, and D. J. Anderson, "A diverse family of GPCRs expressed in specific subsets of nociceptive sensory neurons," Cell, vol. 106, no. 5, pp. 619-632, 2001.

[12] P. M. C. Lembo, E. Grazzini, T. Groblewski et al., "Proenkephalin A gene products activate a new family of sensory neuronspecific GPCRs," Nature Neuroscience, vol. 5, no. 3, pp. 201-209, 2002.

[13] F. Simonin and B. L. Kieffer, "Two faces for an opioid peptide And more receptors for pain research," Nature Neuroscience, vol. 5, no. 3, pp. 185-186, 2002.

[14] C. Ndong, A. Pradhan, C. Puma et al., "Role of rat sensory neuron-specific receptor (rSNSR1) in inflammatory pain: Contribution of TRPV1 to SNSR signaling in the pain pathway," PAIN, vol. 143, no. 1-2, pp. 130-137, 2009.

[15] J. Jiang, D. Wang, X. Zhou et al., "Effect of mas-related gene (Mrg) receptors on hyperalgesia in rats with CFA-induced inflammation via direct and indirect mechanisms," British Journal of Pharmacology, vol. 170, no. 5, pp. 1027-1040, 2013.

[16] Y. J. Liu, F. Fang, J. Q. Fang, and et al., "Analgesic Effect and Mechanism of Electroacupuncture on Rats with Chronic Inflammatory Pain," Chinese Journal of Integrated Traditional And Western Medicine, vol. 36, no. 6, pp. 690-695, 2016.

[17] S. K. Mishra, S. M. Tisel, P. Orestes, S. K. Bhangoo, and M. A. Hoon, "TRPV1-lineage neurons are required for thermal sensation," EMBO Journal, vol. 30, no. 3, pp. 582-593, 2011.

[18] K.-W. Lu, C.-K. Hsu, C.-L. Hsieh, J. Yang, and Y.-W. Lin, "Probing the effects and mechanisms of electroacupuncture at ipsilateral or contralateral ST36-ST37 acupoints on CFA-induced inflammatory pain," Scientific Reports, vol. 6, Article ID 22123, 2016.

[19] Y. Sun, J. Zhang, Y. Lei et al., "Activation of spinal MrgCGi-NR2B-nNOS signaling pathway by mas oncogene-related gene $\mathrm{C}$ receptor agonist bovine adrenal medulla 8-22 attenuates bone cancer pain in mice," American Journal of Translational Research, vol. 8, no. 2, pp. 1144-1154, 2016.

[20] S. A. Honan and P. A. McNaughton, "Sensitisation of TRPV1 in rat sensory neurones by activation of SNSRs," Neuroscience Letters, vol. 422, no. 1, pp. 1-6, 2007.

[21] J. Huang, X. Zhang, and P. A. McNaughton, "Modulation of temperature-sensitive TRP channels," Seminars in Cell \& Developmental Biology, vol. 17, no. 6, pp. 638-645, 2006.

[22] T. L. Yaksh and T. A. Rudy, "Chronic catheterization of the spinal subarachnoid space," Physiology \& Behavior, vol. 17, no. 6, pp. 1031-1036, 1976.

[23] J.-Y. Du, J.-Q. Fang, Y. Liang, and J.-F. Fang, "Electroacupuncture attenuates mechanical allodynia by suppressing the spinal JNK1/2 pathway in a rat model of inflammatory pain," Brain Research Bulletin, vol. 108, pp. 27-36, 2014.

[24] J. L. McDonald, A. W. Cripps, and P. K. Smith, "Mediators, Receptors, and Signalling Pathways in the Anti-Inflammatory and Antihyperalgesic Effects of Acupuncture," Evidence-Based Complementary and Alternative Medicine, vol. 2015, Article ID 975632, 2015.

[25] P.-T. Xu, Z. Song, W.-C. Zhang, B. Jiao, and Z.-B. Yu, "Impaired translocation of GLUT4 results in insulin resistance of atrophic soleus muscle," BioMed Research International, vol. 2015, Article ID 291987, 2015. 
[26] Y. Dai, T. Moriyama, T. Higashi et al., "Proteinase-Activated Receptor 2-Mediated Potentiation of Transient Receptor Potential Vanilloid Subfamily 1 Activity Reveals a Mechanism for Proteinase-Induced Inflammatory Pain," The Journal of Neuroscience, vol. 24, no. 18, pp. 4293-4299, 2004.

[27] M. De Castro Costa, P. De Sutter, J. Gybels, and J. Van Hees, "Adjuvant-induced arthritis in rats: A possible animal model of chronic pain," PAIN, vol. 10, no. 2, pp. 173-185, 1981.

[28] Y. ZHAO, Y. QIN, T. LIU, and D. HAO, “Chronic nerve injuryinduced Mas receptor expression in dorsal root ganglion neurons alleviates neuropathic pain," Experimental and Therapeutic Medicine, vol. 10, no. 6, pp. 2384-2388, 2015.

[29] X. Xiao, X.-T. Zhao, L.-C. Xu et al., "Shp-1 dephosphorylates TRPV1 in dorsal root ganglion neurons and alleviates CFAinduced inflammatory pain in rats," PAIN, vol. 156, no. 4, pp. 597-608, 2015.

[30] S. S. Choi and B. T. Lahn, "Adaptive evolution of MRG, a neuron-specific gene family implicated in nociception," Genome Research, vol. 13, no. 10, pp. 2252-2259, 2003.

[31] Z. Li, P.-Y. Tseng, V. Tiwari et al., "Targeting human Mas-related G protein-coupled receptor X1 to inhibit persistent pain," Proceedings of the National Acadamy of Sciences of the United States of America, vol. 114, no. 10, pp. E1996-E2005, 2017.

[32] H. J. Solinski, I. Boekhoff, M. Bouvier, T. Gudermann, and A. Breit, "Sensory neuron-specific mas-related gene-X1 receptors resist agonist-promoted endocytosis," Molecular Pharmacology, vol. 78, no. 2, pp. 249-259, 2010.

[33] M. Cai, T. Chen, R. Quirion, and Y. Hong, “The involvement of spinal bovine adrenal medulla 22-like peptide, the proenkephalin derivative, in modulation of nociceptive processing," European Journal of Neuroscience, vol. 26, no. 5, pp. 1128-1138, 2007.

[34] C. Wei, W. Huang, X. Xing, and S. Dong, "Dual effects of [Tyr 6]- $\gamma$ 2-MSH(6-12) on pain perception and in vivo hyperalgesic activity of its analogues," Journal of Peptide Science, vol. 16, no. 9, pp. 451-455, 2010.

[35] S.-Q. He, Z. Li, Y.-X. Chu et al., "MrgC agonism at central terminals of primary sensory neurons inhibits neuropathic pain," PAIN, vol. 155, no. 3, pp. 534-544, 2014.

[36] F. Fang, J. Fang, Z. Liu, and et al., "Effects of Electroacupuncture on Bovine Adrenal Medulla 22 and mRNA Expression of its Receptors in Lumbar Dorsal Root Ganglion in Rats with Chronic Inflammatory Pain," Journal of Zhejiang Chinese Medical University, vol. 37, no. 11, pp. 1271-1288, 2013.

[37] J. B. Davis, J. Gray, M. J. Gunthorpe et al., "Vanilloid receptor-1 is essential for inflammatory thermal hyperalgesia," Nature, vol. 405, no. 6783, pp. 183-187, 2000.

[38] M. J. Gunthorpe, C. D. Benham, A. Randall, and J. B. Davis, "The diversity in the vanilloid (TRPV) receptor family of ion channels," Trends in Pharmacological Sciences, vol. 23, no. 4, pp. 183-191, 2002.

[39] T. Christoph, G. Bahrenberg, J. De Vry et al., "Investigation of TRPV1 loss-of-function phenotypes in transgenic shRNA expressing and knockout mice," Molecular and Cellular Neuroscience, vol. 37, no. 3, pp. 579-589, 2008.

[40] Z. Li, S.-Q. He, Q. Xu et al., "Activation of MrgC receptor inhibits $\mathrm{N}$-type calcium channels in small-diameter primary sensory neurons in mice," PAIN, vol. 155, no. 8, pp. 1613-1621, 2014.

[41] H. Luo, J. Cheng, J.-S. Man, and Y. Wan, "Change of vanilloid receptor 1 expression in dorsal root ganglion and spinal dorsal horn during inflammatory nociception induced by complete
Freund's adjuvant in rats," NeuroReport, vol. 15, no. 4, pp. 655658, 2004.

[42] W. Janson and C. Stein, "Peripheral opioid analgesia," Current Pharmaceutical Biotechnology, vol. 4, no. 4, pp. 270-274, 2003.

[43] J.-P. Jiang, Y. Fu, and Y.-G. Hong, "[Bovine adrenal medulla 22 attenuates hyperalgesia in the early phase of complete Freund's adjuvant-induced inflammation in rats].," Sheng li xue bao : [Acta physiologica Sinica], vol. 63, no. 1, pp. 9-19, 2011.

[44] N. García-Sanz, A. Fernández-Carvajal, C. Morenilla-Palao et al., "Identification of a tetramerization domain in the $\mathrm{C}$ terminus of the vanilloid receptor," The Journal of Neuroscience, vol. 24, no. 23, pp. 5307-5314, 2004.

[45] C. Morenilla-Palao, R. Planells-Cases, N. García-Sanz, and A. Ferrer-Montiel, "Regulated exocytosis contributes to protein kinase C potentiation of vanilloid receptor activity," The Journal of Biological Chemistry, vol. 279, no. 24, pp. 25665-25672, 2004.

[46] K. W. Ho, N. J. Ward, and D. J. Calkins, "TRPV1: A stress response protein in the central nervous system," American Journal of Neurodegenerative Diseases, vol. 1, no. 1, pp. 1-14, 2012.

[47] S. Neumann, J. M. Braz, K. Skinner, I. J. Llewellyn-Smith, and A. I. Basbaum, "Innocuous, not noxious, input activates PKC $\gamma$ interneurons of the spinal dorsal horn via myelinated afferent fibers," The Journal of Neuroscience, vol. 28, no. 32, pp. 79367944, 2008. 


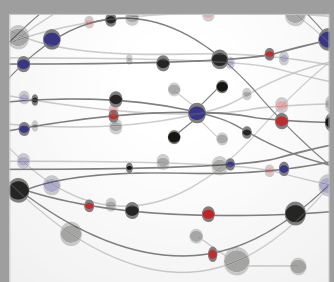

The Scientific World Journal
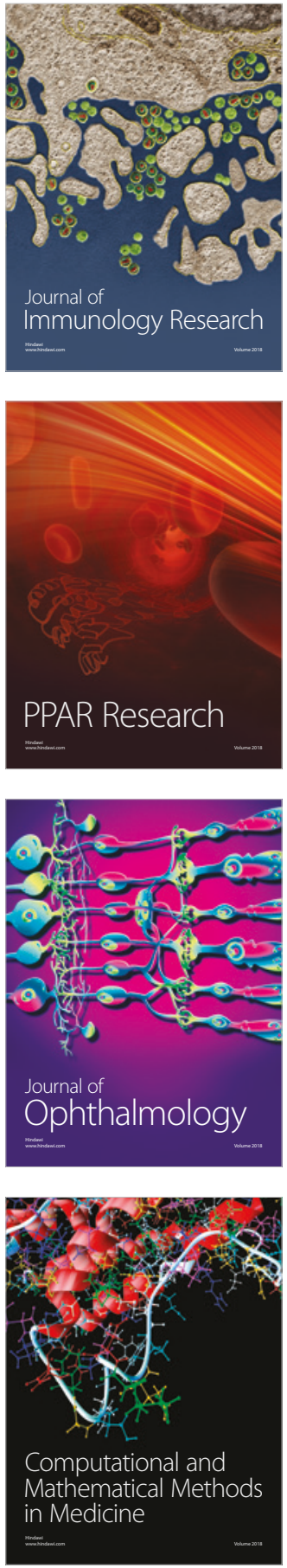

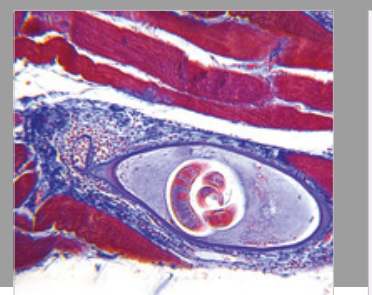

Gastroenterology Research and Practice

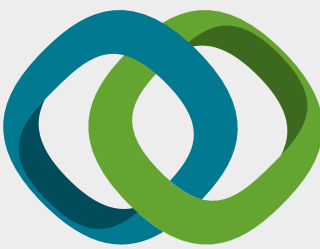

\section{Hindawi}

Submit your manuscripts at

www.hindawi.com
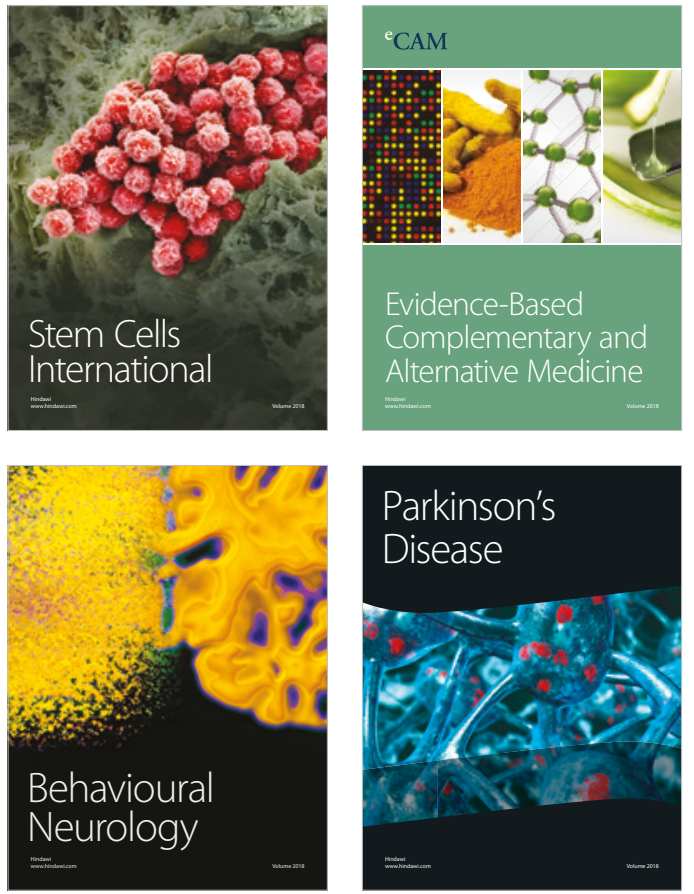

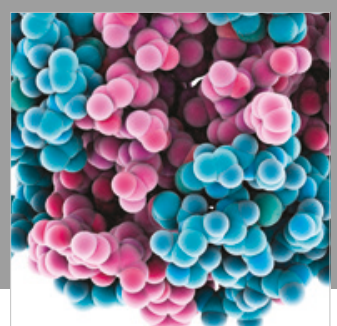

ournal of

Diabetes Research

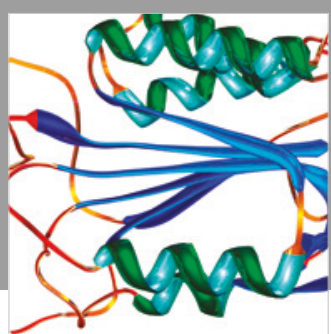

Disease Markers
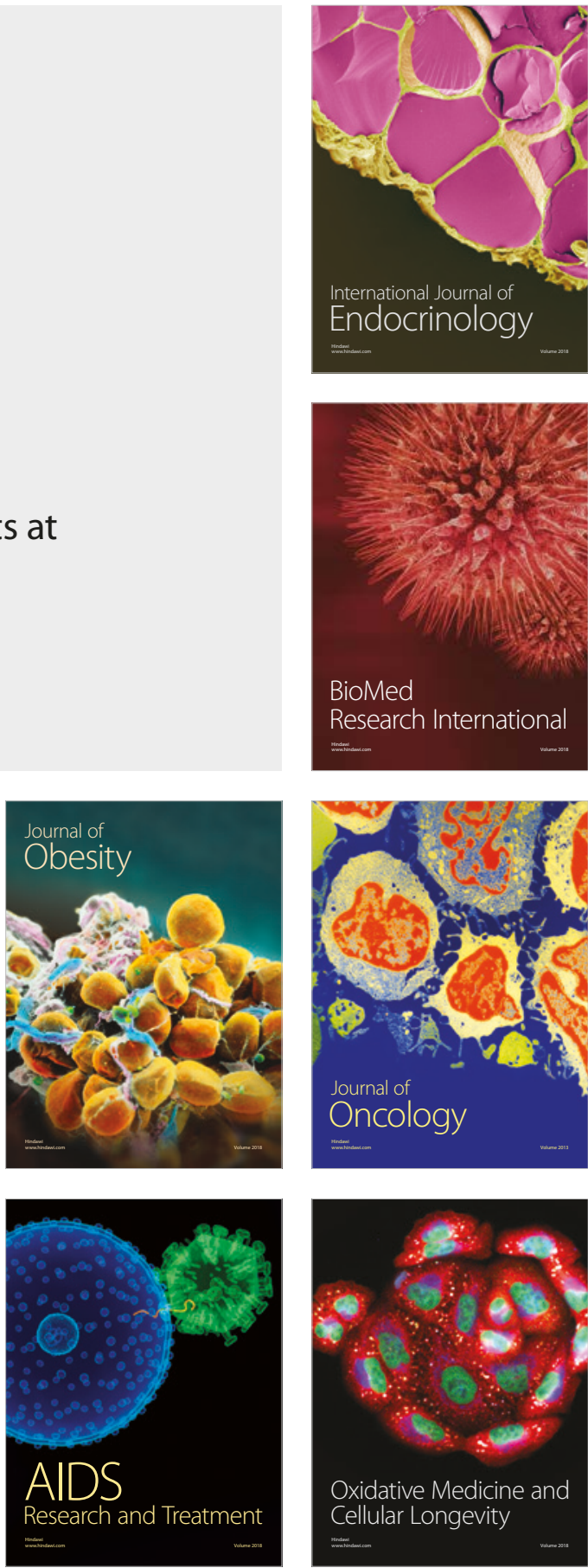